\title{
Enfermedad Pulmonar Obstructiva Crónica y Tabaquismo
}

\author{
FRANCISCO ARANCIBIA H.*
}

\section{Chronic obstructive pulmonary disease and smoking}

Chronic obstructive pulmonary disease (COPD) is a major public health problem worldwide, caused mainly by tobacco consumption. In Chile, the prevalence of COPD is $16.9 \%$ in persons over 40 years of age. In patients with COPD, the prevalence of active smoking is greater and ranges from $29 \%$ to $48 \%$. The cessation of smoking in patients with COPD is the most effective measure to stop the progression of the disease and achieve clinical and spirometric benefits. The treatment of these patients should be mixed, which includes cognitive behavioral therapy (CBT) and pharmacological treatment, because it is more cost-effective.

Key words: Smoking; tobacco use; Pulmonary Disease; Chronic Obstructive; Disease Progression

\section{Resumen}

La enfermedad pulmonar obstructiva crónica (EPOC) es un importante problema de salud pública a nivel mundial, ocasionada principalmente por el consumo de tabaco. En Chile, la prevalencia de la EPOC es de 16,9\% en mayores de 40 años. En pacientes con EPOC la prevalencia de tabaquismo activo es mayor y se encuentra entre el $29 \%$ al $48 \%$. El cese del consumo de tabaco en los pacientes con EPOC es la medida más efectiva para detener la progresión de la enfermedad y lograr beneficios clínicos y espirométricos. El tratamiento de estos pacientes debe ser mixto, en el cual se incluye terapia cognitivo conductual (TCC) y tratamiento farmacológico, por cuanto es más eficaz y con mejor relación costo/efectividad.

Palabras clave: Fumar; tabaquismo; Enfermedad pulmonar obstructiva crónica; progresión de la enfermedad.

\section{Introducción}

La EPOC es una condición patológica crónica respiratoria caracterizada por una persistente limitación del flujo aéreo espiratorio que es parcialmente reversible ${ }^{1}$. La EPOC permanece como uno de los principales problemas de salud pública y es la cuarta causa de mortalidad en Estados Unidos y será la tercera en el año $2020^{2}$.

La EPOC se caracteriza por manifestaciones tanto respiratorias como sistémicas (ansiedad, anemia, reducción de la masa corporal, etc.). ${ }^{3}$ Además, estas enfermedades pueden estar acompañadas de diversas comorbilidades (cardiovascu- lares, respiratorias, metabólicas, depresión, etc. $)^{4}$.

La EPOC es una enfermedad prevenible y tratable, y el factor de riesgo más importante es el tabaquismo, en un $85-90 \%$ de los casos $^{5}$. Otros factores son: la contaminación ambiental, ocupacional e intradomiciliaria, y el déficit de alfa-1- antitripsina ${ }^{6}$.

\section{Epidemiología}

La prevalencia de la EPOC en Santiago de Chile que encontró el estudio Platino, realizado en 5 ciudades de Latinoamérica, fue de $16,9 \%$ en

* Jefe del Servicio de Medicina Instituto Nacional del Tórax.

Profesor agregado, Facultad de Medicina, Universidad de Chile.

Presidente de la Sociedad Chilena de Enfermedades Respiratorias 
Tabla 1. Tratamiento farmacológico para fumadores con EPOC

\begin{tabular}{|c|c|c|c|c|c|c|}
\hline Intervención & [Ref] & Sujetos & Diseño & $\begin{array}{l}\text { Duración } \\
\text { semana }\end{array}$ & Resultado & $\begin{array}{l}\text { Significancia } \\
\text { estadística }\end{array}$ \\
\hline TRN & 18 & $\begin{array}{l}370 \text { fumadores } \\
\text { con EPOC }\end{array}$ & $\begin{array}{l}\mathrm{NS} / \text { placebo, } \\
\text { bajo TCC/ } \\
\text { alto TCC }\end{array}$ & 12 & $\begin{array}{l}\text { TRN:17; } \\
\text { Placebo: } 10 \% \text {; } \\
\text { No diferencias } \\
\text { entre alta y } \\
\text { baja TCC }\end{array}$ & $\begin{array}{l}\text { Si, OR: } 2,88 ; \\
\text { IC: } 95 \% \\
(1,34-6,16)\end{array}$ \\
\hline \multirow{3}{*}{$\begin{array}{l}\text { B u p r o pión } \\
\text { (BP) } \\
\text { y } \\
\text { Nortriptilina } \\
\text { (NT) }\end{array}$} & 25 & $\begin{array}{l}404 \text { fumadores } \\
\text { con EPOC }\end{array}$ & $\mathrm{BP} /$ placebo & 12 & $\begin{array}{l}6 \text { semanas } \\
\text { BP: } 16 \% ; \\
\text { placebo: } 9 \%\end{array}$ & $\begin{array}{l}\mathrm{Si} \\
\mathrm{p}<0,005\end{array}$ \\
\hline & 26 & $\begin{array}{l}255 \\
\text { fumadores con } \\
\text { riesgo EPOC }\end{array}$ & $\begin{array}{l}\mathrm{BP} / \mathrm{NT} / \\
\text { placebo }\end{array}$ & 12 & $\begin{array}{l}6 \text { semanas } \\
\text { BP:28\%; NT: } \\
25 \% \text {; placebo: } \\
15 \%\end{array}$ & $\begin{array}{l}\text { Si para BP vs } \\
\text { placebo, } \\
\text { No para NT vs } \\
\text { placebo }\end{array}$ \\
\hline & 30 & $\begin{array}{l}255 \text { fumado- } \\
\text { res con riesgo } \\
\text { EPOC }\end{array}$ & $\begin{array}{l}\mathrm{BP} / \mathrm{NT} / \\
\text { placebo }\end{array}$ & 12 & $\begin{array}{l}12 \text { semanas } \\
\text { BP: } 21 \% \text {; } \\
\text { NT: } 20 \% \text {; } \\
\text { placebo: } \\
13,5 \%\end{array}$ & No \\
\hline $\begin{array}{l}\text { Vareniclina } \\
(\mathrm{VRN})\end{array}$ & 23 & $\begin{array}{l}504 \text { fumadores } \\
\text { con EPOC }\end{array}$ & VRN/placebo & 12 & $\begin{array}{l}12 \text { semanas } \\
\text { VRN: } 18,6 \% \text {; } \\
\text { placebo: } 5,6 \%\end{array}$ & $\begin{array}{l}\mathrm{Si} \text {, } \\
\text { OR: } 4,04 \mathrm{IC} \\
95 \%\end{array}$ \\
\hline
\end{tabular}

La tabla sólo muestra ensayos aleatorios, doble ciego, controlados con placebo. TRN: terapia de reemplazo de nicotina; BN: Bupropión; NS: nicotina sublingual; TCC: terapia cognitivo conductual; VRN; vareniclina.

mayores de 40 años, con un importante subdiagnóstico ${ }^{7}$. No obstante, el porcentaje de pacientes con diagnostico de EPOC que continúa fumando, es elevado. En efecto en 4 estudios prospectivos multicéntricos realizados en pacientes con EPOC, se encontró una prevalencia de $29,3 \%$ a $48 \%$ de tabaquismo activo ${ }^{8-11}$. En otro estudio realizado en Inglaterra, se encontró que la prevalencia del tabaquismo aumentaba con la gravedad de la EPOC $^{12}$. Por tanto, parece de mucha importancia lograr el cese del consumo de tabaco en los pacientes con EPOC, por cuanto es la única medida que se ha mostrado ser eficaz para detener la evolución progresiva de esta enfermedad ${ }^{13}$. Desafortunadamente, el cese del consumo de tabaco en estos pacientes parece más difícil y se atribuye a que tienen características especificas que reducen su éxito ${ }^{14-15}$. Estos pacientes tienen: mayor grado de dependencia nicotínica (Fagerström $>$ 7), niveles más altos de monóxido de carbono en aire expirado, mayor inhalación y depósito de sustancias tóxicas del humo del tabaco; baja motivación y percepción de autoeficacia, baja expectativa de mejorar, mayor preocupación por el aumento de peso y una alta prevalencia de comorbilidad psiquiátrica (depresión).

\section{Cese del consumo de tabaco}

El cese del consumo de tabaco tiene muchos beneficios y en los pacientes con EPOC es la medida mas efectiva para controlar la progresión de la enfermedad, reducir la declinación anual del $\mathrm{VEF}_{1}$, mejorar la respuesta a los medicamentos broncodilatadores y corticoides inhalados y reducir la incidencia de exacerbaciones agudas e infecciones bronquiales ${ }^{14,16}$.

Además de los beneficios antes mencionados de la cesación del tabaquismo, el estudio de Kanner et al., y el de Tonnesen et al., demostraron que una intervención para dejar de fumar en pacientes con EPOC disminuyo los síntomas como: disnea, tos, producción de esputo y sibilancias $^{17,18}$. La cesación tabáquica es recomendada para todos los pacientes con EPOC independiente del estadio de la enfermedad. Los síntomas respiratorios pueden mejoran después de $3-9$ meses de haber dejado de fumar y la función pulmonar puede aumentar en un $10 \%$ según el estudio de Barnett et al ${ }^{19}$.

El riesgo de desarrollar EPOC disminuye en alrededor de la mitad con el abandono del taba$\mathrm{co}^{20}$. 


\section{Tratamiento de cesación del tabaquismo}

Recientemente, han sido publicadas las recomendaciones de la SEPAR y de la ERS para el abandono del tabaquismo en fumadores con EPOC $^{16,20}$. En ambas se recomienda que el tratamiento del tabaquismo en fumadores con EPOC debe ser mixto, en el cual se incluye terapia cognitivo conductual (TCC) y tratamiento farmacológico, por cuanto es más eficaz y con mejor relación costo/efectividad ${ }^{22}$.

La oportunidad para intervenir en estos pacientes es en todo contacto con el sistema de salud y en particular la hospitalización. En estos pacientes es importante conocer el grado de tabaquismo, el grado de dependencia, grado de motivación y autoeficacia y si presenta depresión ${ }^{16}$. El asesoramiento a estos pacientes que recomienda la ERS incluye: 1) Explicación clara de la relación entre el tabaquismo y la EPOC, y de la relación entre el abandono del tabaco y la mejoría de la EPOC; 2) Uso de los resultados espirométricos, monitorizar niveles de $\mathrm{CO}$ y la edad pulmonar para aumentar la motivación para dejar de fumar; 3) Establecer una fecha para dejar de fumar, identificar situaciones de alto riesgo y desarrollar habilidades de afrontamiento; 4) Organizar visitas de seguimiento para abordar específicamente el abandono de esta adicción; 5) Explicación del síndrome de abstinencia; 6) Proporcionar materiales de autoayuda y 7) Envío de cartas personales, correos electrónicos y mensajes de texto.

En cuanto al tratamiento farmacológico, vareniclina es un fármaco de primera línea para el tratamiento del tabaquismo en pacientes con EPOC, independientemente de la gravedad de la enfermedad o el número de cigarrillos fumados. En 2 estudios realizado con vareniclina, para ayudar a dejar de fumar a pacientes con EPOC, demuestran eficacia y seguridad de su uso a los 12 meses de seguimiento ${ }^{13,23}$. Recomendación con calidad de evidencia alta. Nivel de recomendación: fuerte.

En cuanto al bupropión, es una ayuda eficaz y seguro de usar para apoyar la cesación del tabaquismo en los pacientes con EPOC. Dos estudios clínicos demostraron que es más eficaz que placebo y la seguridad en obtener abstinencia continua a los 6 meses de seguimiento ${ }^{24,25}$. Recomendación consistente con una calidad de evidencia alta. $\mathrm{Ni}$ vel de recomendación: fuerte. Si se utiliza vareniclina o bupropión, se recomienda prolongar hasta 6 meses el tratamiento en aquellos fumadores que han utilizado este fármaco previamente a dosis estándar y no han tenido éxito o que estando utilizando a dosis estándar no controlan los síntomas del síndrome de abstinencia.

La terapia de reemplazo con nicotina (TRN), en distintos estudios ha mostrado eficacia y seguridad para ayudar a dejar de fumar a los pacientes con EPOC ${ }^{13,26,27}$. Recomendación consistente con una calidad de evidencia alta. Nivel de recomendación: fuerte. Sin embargo, la dosis estándar de TRN en las poblaciones de EPOC han producido índices de abandono más bajos que en la población general de fumadores (Nivel de Evidencia A) ${ }^{28}$. Por ello, se recomienda una dosis alta de TRN en los pacientes que informan niveles moderados a altos de adicción a la nicotina (test de Fagerstrôm). La combinación de dos tipos de TRN con diferentes tipos de liberación (ejemplo parches más chicles) es altamente recomendable (Nivel de Evidencia A).

El aumento de la duración de la TRN hasta seis o doce meses puede ser eficaz para aumentar las tasas de abstinencia en comparación con las 10 semanas estándar de la terapia con TRN (Nivel de Evidencia A) ${ }^{28}$.

La medición de monóxido de carbono $(\mathrm{CO})$ de aire exhalado y la cotinina (pelo, orina ) son biomarcadores no invasivos útiles de la exposición al tabaquismo y pueden utilizarse para evaluar el tabaquismo y controlar el abandono del tabaco (Nivel de Evidencia A) ${ }^{28}$.

\section{Conclusiones}

La prevalencia de tabaquismo activo en los pacientes con EPOC es elevada y la cesación de esta adicción es la medida más efectiva para detener la progresión de la enfermedad, mejorar los síntomas, la respuesta a los medicamentos, y reducir las exacerbaciones agudas. El tratamiento de estos pacientes debe incluir una combinacion de terapia cognitivo conductual y tratamiento farmacológico y es esencial realizar el ABC-D en todos estos pacientes como primera aproximación.

\section{Bibliografía}

1.- VANFLETEREN LE, SPRUIT MA, WOUTERS EF, FRANSSEN FM. Management of chronic obstructive pulmonary disease beyond the lungs. Lancet Respir Med 2016; 4: 911-24.

2.- MURRAY C, LÓPEZ A. Alternative projections of mortality and disability by cause 1990-2020: Global burden of Disease Study. Lancet 1997; 349: 1498-504.

3.- BRACKE KR, D'HULST AI, MAES T, MOERLOOSE KB, DEMEDTS IK, LEBECQUE S, et al. 
Cigarette smoke-induced pulmonary inflammation and emphysema are attenuated in CCR6-deficient mice. J Immunol. 2006; 177: 4350-9.

4.- Global Initiative for Chronic Obstructive Lung Disease (GOLD). Global strategy for Diagnosis, Management and Prevention of Chronic Obstructive Pulmonary Disease. 2011. Arch Bronconeumol. 2013; 49: 354-63.

5.- VESTBO J, HURD SS, AGUSTÍ AG, JONES PW, VOGELMEIER C, ANZUETO A, et al. Global strategy for the diagnosis, management, and prevention of chronic obstructive pulmonary disease: GOLD executive summary. Am J Respir Crit Care Med. 2013; 187 : 347-65.

6.- MENEZES AM, PÉREZ-PADILLA R, JARDIM JR, MUINO A, LÓPEZ MV, VALDIVIA G, et al. Chronic obstructive pulmonary disease in five Latin American cities (the PLATINO study): A prevalence study. Lancet 2005; 366: 1875-81.

7.- WEDZICHA JA, BANERJI D, CHAPMAN KR, VESTBO J, ROCHE N, AYERS RT, et al. Indacaterol-Glycopyrronium versus Salmeterol-Fluticasone for COPD. N Engl J Med 2016; 374: 2222-34.

8.- CALVERLEY PM, ANDERSON JA, CELLI B, FERGUSON GT, JENKINS C, JONES PW, et al. Salmeterol and fluticasone propionate and survival in chronic obstructive pulmonary disease. N Engl J Med 2007; 356: 775-89.

9. TASHKIN DP, CELLI B, SENN S, BURKHART D, KESTEN S, MENJOGE S, et al. A 4-year trial of tiotropium in chronic obstructive pulmonary disease. $\mathrm{N}$ Engl J Med. 2008 Oct 9; 359: 1543-54.

10. LARSSON K, JANSON C, LISSPERS K, JØRGENSEN L, STRATELIS G, TELG G, et al. Combination of budesonide/formoterol more effective than fluticasone/salmeterol in preventing exacerbations in chronic obstructive pulmonary disease: the PATHOS study. J Intern Med. 2013; 273: 584-94.

11.- SHAHAB L, JARVIS MJ, BRITTON J, WEST R. Prevalence, diagnosis and relation to tobacco dependence of chronic obstructive pulmonary disease in a nationally representative population sample. Thorax 2006; 61: 1043.7.

12.- ANTHONISEN NR, CONNETT JE, KILEY JP, ALTOSE MD, BAILEY W, BUIST S, et al. Effects of smoking intervention and the use of an inhaled anticholinergic bronchodilator on the rate of decline of FEV1: The Lung Health Study. JAMA 1994; 272: 1497-505.

13.- JIMÉNEZ RUIZ C, RAMOS PINEDO A, CICERO GUERRERO A, MAYAYO ULIBARRI M, CRISTOBAL FERNÁNDEZ M, LÓPEZ GONZÁLEZ G. Characteristics of COPD smokers and effectiveness and safety of smoking cessation medications. Nicotine Tob Res. 2012; 14: 1035-9.

14. MASEFIELD S, POWELL P, JIMÉNEZ-RUIZ C, HAJEK P, LEWIS K, ANDREAS S, et al. Recommendations to improve smoking cessation outcomes from people with lung conditions who smoke. ERJ Open Res 2016; 2: 00009-2016.

15.- JIMÉNEZ-RUIZ CA, RIESCO MIRANDA JA, ALTET GÓMEZ N, LORZA BLASCO JJ, SIGNESCOSTA MIÑANA J, SOLANO REINA S, et al. Sociedad Española de Neumología y Cirugía Torácica (SEPAR). Treatment of smoking in smokers with chronic obstructive pulmonary disease. Sociedad Española de Neumología y Cirugía Torácica (SEPAR). Arch Bronconeumol. 2013; 49: 354-63.

16.- KANNER RE, CONNETT JE, WILLIAMS DE, BUIST AS. Effects of randomized assignment to a smoking cessation intervention and changes in smoking habits on respiratory symptoms in smokers with early chronic obstructive pulmonary disease: the Lung Health Study. Am J Med 1999; 106: 410-6.

17.- TØNNESEN P, MIKKELSEN K, BREMANN L. Nurse-conducted smoking cessation in patients with COPD using nicotine sublingual tablets and behavioral support. Chest 2006; 130: 334-42.

18.- BARNETT M. COPD: Smoking cessation Community Nursing Journal 2008; 22: 36-40.

19.- LANIADO-LABORÍN R. Smoking and Chronic Obstructive Pulmonary Disease (COPD). Parallel Epidemics of the 21st Century. Int J Environ Res Public Health 2009; 6: 209-24.

20.- JIMÉNEZ-RUIZ CA, ANDREAS S, LEWIS KE, TONNESEN P, VAN SCHAYCK CP, HAJEK P, et al. Statement on smoking cessation in COPD and other pulmonary diseases and in smokers with comorbidities who find it difficult to quit. Eur Respir J 2015; 46: 6179.

21.- HOOGENDOORN M, FEENSTRA TL, HOOGENVEEN RT, RUTTEN-VAN MÖLKEN MPMH. Long term effectiveness and cost-effectiveness of smoking cessation interventions in patients with COPD. Thorax 2010; 65: 711-8.

22.- TASHKIN DP, RENNARD S, HAYS JT, LAWRENCE D, MARTON JP, LEE TC. Effects of varenicline on smoking cessation in patients with mild to moderate COPD: A randomized controlled trial. Chest 2011; 139: 591-9.

23.- TASHKIN DP, KANNER R, BAILEY W, BUIST S, ANDERSON P, NIDES M, et al. Smoking cessation in patients with chronic obstructive pulmonary disease: A doubleblind, placebo-controlled, randomised trial. Lancet 2001; 357: 1571-5.

24.- WAGENA EJ, KNISPCHILD PG, HUIBERS MJ, WOUTERS EF, VAN SCHAYK CP. Efficacy of bupropión and nortriptyline for smoking cessation among people at risk for or with COPD. Arch Intern Med 2005; 165: 2286-92.

25.- STRASSMANN R, BAUSCH B, SPAAR A, KLEIJNEN J, BRAENDLI O, PUHAN MA. Smoking cessation interventions in COPD: A network meta-analysis of randomised trials. Eur Respir J 2009; 34: 634-40. 
26.- SUNDBLAD BM, LARSSON K, NATHELL L. High rates of smoking abstinence in COPD patients: Smoking cessation by hospitalization. Nicotine Tob Res 2008; 10 : 883-90.

27.- TAYLOR H. Pharmacological Interventions for Smoking Cessation in COPD Patients. En eds. Behrakis Pk, Vardavas C, Papadakis S. Tobacco Cessation Guidelines for High-Risk Populations. European Union's Health Programme 2017: 225-32.

28.- VAN SCHAYCK CP, KAPER J, WAGENA EJ, WOUTERS EF, SEVERENS JL. The cost-effectiveness of antidepressants for smoking cessation in chronic obstructive pulmonary disease (COPD) patients. Addiction 2009; 104: 2110-7. 